\title{
Antibiotic resistance profiling of clinical isolates of Salmonella enterica Serovar Paratyphi A in Dhaka, Bangladesh
}

\author{
Md. Mobarok Hossain ${ }^{1}$, Tasnim Jabin ${ }^{1}$, Md. Ifrat Hossain ${ }^{2}$, Mst. Arzina Khatun ${ }^{2}$, Md. Hossain \\ Emam $^{2}$, Muhammad Asaduzzaman ${ }^{2}$ and Md. Aftab Uddin² \\ ${ }^{1}$ Department of Genetic Engineering and Biotechnology, University of Chittagong, Bangladesh \\ ${ }^{2}$ Department of Microbiology, Stamford University Bangladesh, 51, Siddeswari Road, Dhaka-1217, Bangladesh
}

\author{
Received 03 August 2021/Accepted 23 September 2021
}

\begin{abstract}
The study was conducted to detect the antibiotic resistance profile of the clinical isolates of Salmonella enterica Serovar Paratyphi A from 100 blood samples of patients from different age groups suspected to be suffering from enteric fever. The pure cultures of the bacterial isolates were collected from some renowned diagnostic centers of Dhaka and they were further characterized through the conventional culture, microscopy and biochemical examinations. These isolates were cross checked for the antibiogram profile by the Kirby-Bauer disc diffusion method against ten different types of antibiotics. Most of the isolates were found resistant against azithromycin $(100 \%)$, nalidixic acid (100\%) and ceftazidime (75\%). However, isolates showed sensitivity to ciprofloxacin (95\%), levofloxacin $(97 \%)$, cotrimoxazole $(96 \%)$ and chloramphenicol $(95 \%)$. These findings highlight the need for continuous monitoring of the drug resistance pattern of $S$. enterica Serovar Paratyphi A for better public health management.
\end{abstract}

Keywords: Paratyphi, Antibiogram Profiling, Resistant, Sensitive, Enteric Fever.

\section{INTRODUCTION}

Enteric fever is a type of intestinal tract disease caused by Salmonella enterica serovars Typhi (producing typhoid fever) and Paratyphi (paratyphoid fever) due to which patients may experience fever, malaise, abdominal pain and constipation (1). This is a major public health concern in many developing countries. More than $90 \%$ of typhoid fever cases in terms of morbidity and mortality were reported in South East Asia in the recent past (2). Symptoms of paratyphoid fever, usually express 6-30 days after exposure. Often, a steady onset of a high fever occurs over several days. Weakness, loss of appetite, and headaches are also normally experienced. Without treatment, symptoms may last for weeks or months (3). A skin rash with rose-colored spots may also develop in some cases (4). The carriers are capable of transmitting disease to others (5). The etiological agent of paratyphoid fever is usually spread by eating or drinking food or water contaminated with the feces of an infected person (3). This may occur when an infected person is engaged in preparation the food items (4). Poor sanitation is considered as the major risk factor found among poor crowded populations (6). Sporadically, the disease may spread through sexual contact where human is the only animal known to contract such infection (3). Isolation of the organism from the blood culture is crucial for the diagnosis (7).

Antibiotic resistance creates a noteworthy risk in terms of mortality and economic burden throughout the world. However, the developing countries are more affected because of the widespread and inappropriate use of antibiotics. Use of non-human antibiotics may reduce the performance of drugs. A number of factors are associated for this situation such as underprivileged healthcare standards, malnutrition, chronic and recurring infections, and inability to afford more effectual and expensive drugs. (8). Furthermore, the shortage of novel and effective drugs indicate that resistance must be restricted before we run out of choices to combat it. Bangladesh is regarded to have the maximum degree of antibiotics resistance that may pose a regional and threat to the treatment of infectious diseases (8).

Ampicillin, chloramphenicol, and co-trimoxazole are considered as the first-line antibiotics for the treatment of enteric fever (9). The reports on multidrug-resistant strains of Salmonella have been available since the late 1980s (10). As per the guiding principle of the World Health Organization, fluoroquinolone is the drug of preference if the etiological agent is multi-drug resistant (MDR) and quinolone sensitive. This has directed to the enhanced use of third-generation cephalosporins since the widespread emergence of Salmonella spp. isolates that are multi drug-resistant to the more usual antimicrobial drugs (1). The practitioners generally have no common consensus regarding the selection of drugs for the treatment of enteric fever. This prompted us to conduct the present study with the aim to observe the current trend in the sensitivity pattern of S. enterica Serovar Paratyphi A to different groups of

*Corresponding Author: Mailing address. Md. Aftab Uddin, Assistant Professor, Department of Microbiology, Stamford University Bangladesh, 51 Siddeswari Road, Dhaka-1217, Bangladesh; E-mail: aftab_mb12@stamforduniversity.edu.bd. 
antibiotics commonly prescribed in Bangladesh.

\section{MATERIALS AND METHODS}

Sample collection. Venous blood samples were collected from patient suspected to be suffering from paratyphoid fever between October 2020 and December 2020. Each tube was carefully labeled with the sample number. The samples were collected from various diagnostic centers of Dhaka city, Bangladesh.

Bacterial isolation. The blood samples were aseptically transferred to blood culture broth (Hi-Media, India) and incubated overnight at $37^{\circ} \mathrm{C}$ for 18 to 24 hours. Tubes that showed turbidity were sub-cultured on Xylose Lysine Deoxycholate agar (Hi-Media, India) at $37^{\circ} \mathrm{C}$ and were observed for the presence of red colonies with black centers for the suspected of Salmonella spp. isolates (11)

Microscopic analysis. Microscopic analysis of the isolates was done for the determination of bacterial size, shape and staining properties by gram staining (11). Cultural and morphological characteristics were identified following standard microbiological procedures (12).

Biochemical tests. Recommended biochemical tests were performed to validate the identification of all the pathogenic isolates found from blood samples by the formerly described method (12)

Study of Antibiogram. Kirby-Bauer method was applied for the antibiotic susceptibility assay (13). Drug sensitivity pattern was observed agains Ampicillin $(10 \mu \mathrm{g})$, Azithromycin $(15 \mu \mathrm{g})$, Cefixime $(5 \mu \mathrm{g})$, Ceftazidime (30 $\mu \mathrm{g})$, Cefuroxime $(30 \mu \mathrm{g})$, Chloramphenicol $(30 \mu \mathrm{g})$, Ciprofloxacin $(5 \mu \mathrm{g})$, Nalidixic Acid $(30 \mu \mathrm{g})$, Cotrimoxazole $(30 \mu \mathrm{g})$ and Levofloxacin $(30 \mu \mathrm{g})$. Bacteria were categorized as resistant, intermediate or susceptible to each antibiotic disc (Hi-Media, India) used according to standard CLSI guideline 2013 (14)

\section{RESULTS AND DISCUSSION}

Salmonella Paratyphi A appears to be responsible for a substantial proportion of enteric fever cases in many Asian countries, sometimes accounting for $50 \%$ of
Salmonella isolates in blood stream among enteric fever patient $(15,16)$. The susceptibility pattern of antimicrobials against $S$. enterica Serovar Paratyphi A was conducted in this study. This study highlighted the need for surveillance of antibiotic susceptibility pattern of the suspected bacterial isolates with a view of selecting precise antibiotic therapy in order to avoid the development of drug resistance. The study was conducted to assess the antibiotic resistance pattern of $S$. enterica Serovar Paratyphi A after investigating 100 clinically suspected patients suffering from paratyphoid fever. In our analysis, all suspected isolates were examined for the presence of Salmonella spp. red colonies with black centers on XLD agar. Isolates were then confirmed by Gramstaining microscopic analysis and distinctive kinds of biochemical tests (Table 1)

In our investigation, a total of 100 male and female patients who were suspected to be suffering from typhoid fever in different age groups from 1 to 56 years old (Figure 1a). We found that male $(n=68$, $68 \%$ ) were likely to be more susceptible to paratyphoid infection than female patients $(n=32$, 32\%) (Figure 1b).

One hundred clinical isolates of S. enterica Serovar Paratyphi A were subjected to antimicrobial susceptibility tests against different commercial antibiotics. Isolates showed $100 \%$ resistance against

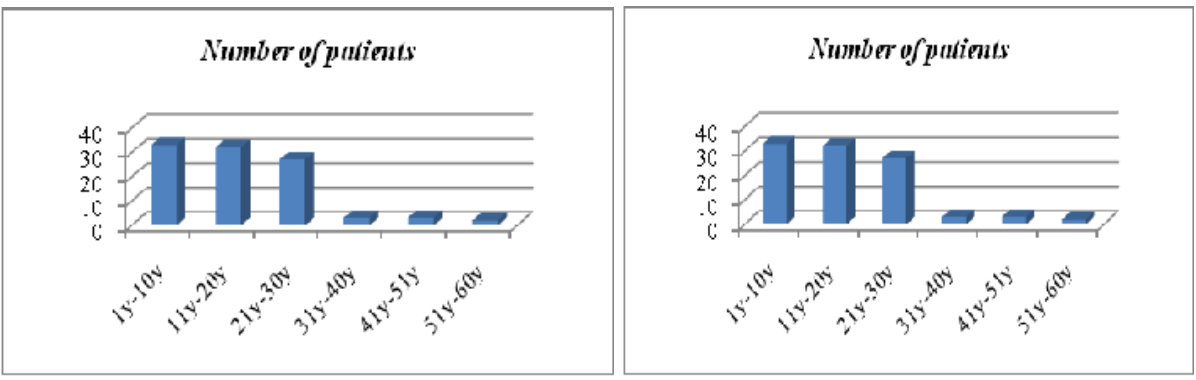

Figure 1. In the left, distribution of age groups among respondents (a), and in the right, distribution of gender among respondents (b).

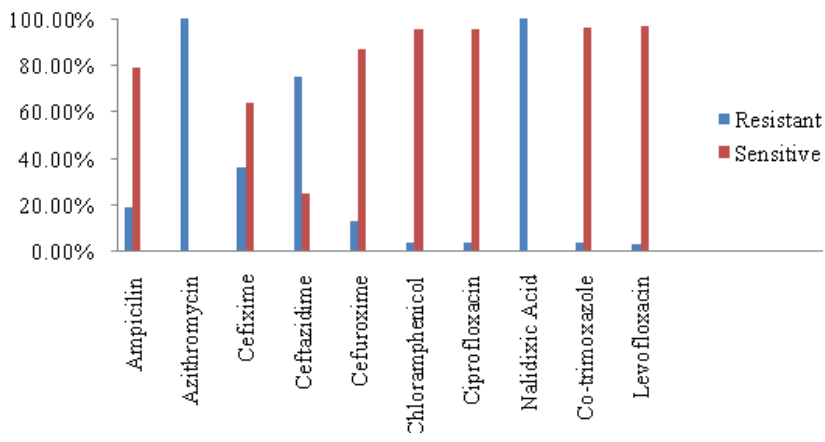

Figure 2. Antibiotic resistance pattern of the bacterial isolates $(\mathrm{n}=100)$.

Table 1. Biochemical tests for the detection of S. enterica Serovar Paratyphi A.

\begin{tabular}{|c|c|c|c|c|c|c|c|c|c|c|}
\hline $\begin{array}{l}\text { Assumed } \\
\text { organism }\end{array}$ & $\begin{array}{r}\mathrm{TS} \\
\text { Slant/B }\end{array}$ & Tas & Indole & MR & VP & Citrate & Oxidase & Catalase & $\begin{array}{c}\text { Sugar } \\
\text { Fermentation }\end{array}$ & $\begin{array}{c}\text { Nitrate } \\
\text { Reduction }\end{array}$ \\
\hline Salmonella spp. & $\begin{array}{ll}\mathrm{R} & \mathrm{Y}\end{array}$ & + & - & + & - & - & - & - & - & + \\
\hline
\end{tabular}


azithromycin and nalidixic acid, followed by ceftazidime $(75 \%)$, cefixime $(36 \%)$, ampicillin $(18.83 \%)$ and cefuroxime (13\%), However, ciprofloxacin (95.83\%). levofloxacin (97\%), cotrimoxazole (96\%) and chloramphenicol $(95.83 \%)$ were found to be effective drugs against most of the isolated microorganisms according to the CLSI guideline (Figure 2).

These findings were in agreement with our previous study (17). Some authors also reported the growing resistance trend of ciprofloxacin against the pathogens causing enteric fever $(18,19)$. These results are in agreement with previous studies $(20,21)$. In the recent study, most of the isolates were sensitive to ciprofloxacin and a few of them showed the resistance against this antibiotic. Resistance to nalidixic acid has also been reported in India, China and Tunisia (22). Implementing proper rules and regulations on antibiotic use as well as training clinicians and healthcare professionals can abate the uncontrolled use of antibiotics. However, more research need to be conducted for making a better interpretation of our current findings. Therefore, surveillance process needs to be strengthened so that appropriate antibiotics can be applied decrease the rate of resistance.

\section{CONCLUSION}

The emergence of antimicrobial resistance in any part of the world may have global implications and is, therefore a concern for all. Physicians should reconsider before prescribing the antibiotics, in particular nalidixic acid and azithromycin. Based on findings of current study, we recommend appropriate initiatives to monitor and control the use of antibiotics, as well as nationwide surveillance of infectious agents following standardized methodologies.

\section{ACKNOWLEDGEMENT}

We thank the Department of Microbiology, Stamford University Bangladesh, for the necessary technical and laboratory support throughout our study.

\section{REFERENCES}

1. Sharvani R, Hemavathi, Dayanand DK, Shenoy P and Sarmah P.
2016. Antibiogram of Salmonella isolates: time to consider antibiotic salvage. J. Clin. Diagn. Res. 10(5):6-8

2. Hardjo Lugito NP and Cucunawangsih. 2017. Antimicrobial Resistance of Salmonella enterica Serovars Typhi and Paratyphi Isolates from a General Hospital in Karawaci, Tangerang, Indonesia: A Five-Year Review. Int. J. Microbiol. 2017:6215136.

3. Anna EN. 2014. Infectious Diseases Related To Travel. CDC health information for international travel, the yellow book.

4. Jeremy H, Norman B, Iain B, Ralf R, Julius W and Karl E. 2012. Communicable disease control and health protection handbook (3rd ed.). Chichester, West Sussex, UK: Wiley-Blackwell.

5. Edward R, David H, Tom S and Alan M. 2013. Hunter's tropical medicine and emerging infectious diseases $\left(9^{\text {th }}\right.$ ed.). London: Saunders/Elsevier. 568-572.

6. Crump JA and Mintz ED. 2010. Global trends in typhoid and paratyphoid Fever. Clin. Infect. Dis. 50(2):241-6.

7. Chang MS, Woo JH and Kim S. 2019. Management of typhoid fever clinical and historical perspectives in Korea. Infect. Chemother. 51:330-335.

8. Ahmed I, Rabbi MB and Sultana S. 2019. Antibiotic resistance in Bangladesh: A systematic review. Int. J. Infect. Dis. 80:54-61.

9. Britto CD, Wong VK, Dougan G and Pollard AJ. 2018. A systematic review of antimicrobial resistance in Salmonella enterica serovar Typhi, the etiological agent of typhoid. Plos Negl. Trop. Dis. 12(10):e006779.

10. Shrestha KL, Pant ND, Bhandari R, Khatri S, Shrestha B and Lekhak B. 2016. Re-emergence of the susceptibility of the Salmonella spp. isolated from blood samples to conventional first line antibiotics. Antimicrob. Resist. Infect. Control 5:22.

11. Bergey DH, Krieg NR and Holt JG. 1984. Bergey's Manual of Systematic Bacteriology. $6^{\text {th }}$ ed. East Preston Street, Baltimore. 161172

12. Collee JG, Marmion BP and Fraser AJ. 1996. Practical Medical Microbiology. 14th ed. Churchill Livingstone, New York. 13149,166-7.

13. Bauer AW, Kirby W, Sheris JC and Truck M. 1966. Antibiotic susceptibility testing by a standardized single disc method. Am. J. Clin. Path. 145:225-230.

14. Clinical and Laboratory Standards Institute. 2013. Performance Standards for Antimicrobial Susceptibility Testing. Twenty-third Informational Supplement M100-S23. CLSI, Wayne, PA, USA.

15. Shaw AV, Reddy EA and Crump JA. 2008. Etiology of communityacquired bloodstream infections in Africa. 46th Annual Meeting of the Infectious Diseases Society of America; Washington, DC: Infectious Diseases Society of America.

16. Ochiai RL, Wang XY, von Seidlein L, Yang J, Bhutta ZA, Bhattacharya SK et al. Salmonella Paratyphi A rates, Asia. Emerg. Infect. Dis. 11:1764-6.

17. Jabin T, Siam EA, Dipu MR, Asaduzzaman M and Uddin A. 2021. Phenotypic characterization of Salmonella typhi from clinical specimens and determination of the drug susceptibility pattern of the isolates. Iran. J. Med. Microbiol. 15(2):189-194.

18. Rahman A, Ahmed M, Begum R, Ghosh A and Hossain M. 2008. Multidrug resistant typhoid fever in children: a review. J. Dhaka Med. Coll. 17:121-126.

19. Radji M, Fauziah S and Aribinuko N. 2011. Antibiotic sensitivity pattern of bacterial pathogens in the intensive care unit of Fatmawati Hospital, Indonesia. Asian Pac. J. Trop. Biomed. 1:39-42.

20. Akter L, Hassan M and Ahmed Z. 2012. Present status and antibiotic sensitivity pattern of Salmonella typhi and S. paratyphi in different age group hospitalized patients in Dhaka city, Bangladesh. IOSR J. Pharm. Biol. Sci. 4:27-30.

21. Ahmed D, D'Costa LT, Alam K, Nair GB and Hossain MA. 2006. Multidrug-resistant Salmonella enterica serovar typhi isolates with high-level resistance to ciprofloxacin in Dhaka, Bangladesh. Antimicrob. Agents Chemother. 50:3516-3517.

22. Sun L, Klein EY and Laxminarayan R. 2012. Seasonality and temporal correlation between community antibiotic use and resistance in the United States. Clin. Infect. Dis. 55:687-694. 